\title{
ECESARIO A POSTERIORI
}

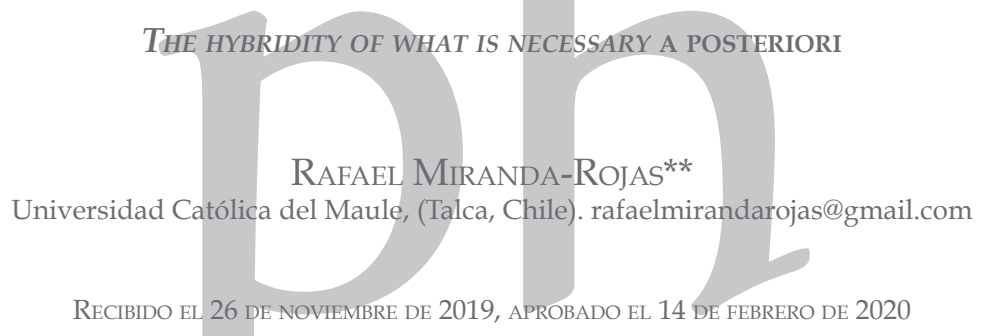

\section{Resumen Abstract}

El presente escrito propone que lo necesario a posteriori corresponde a un caso híbrido de justificación, sin que ello conduzca a un compromiso racionalista extremo. En los casos de enunciados necesarios a posteriori, los principios lógicos que instancian son de carácter a priori, y la información de enunciados como "Agua es $\mathrm{H}_{2} \mathrm{O}^{\prime}$ o "El dolor es la estimulación de la fibra - c" son cognoscibles solo a posteriori. Para explicar esta hibridez, se discutirá cómo la estrategia vía reductio permite aceptar una aproximación racionalista moderada. Se considerará el bootstrapping como caso paradigmático de justificación epistémica híbrida. La discusión respecto al nexo $a$ priori - a posteriori tiende a establecer una jerarquía, en la que uno de los accesos epistémicos es privilegiado, lo que se rechazará.

\section{Palabras clave}

Necesidad, a posteriori, a priori, concebible, posible.
This paper proposes that what is necessary a posteriori corresponds to a hybrid case of justification, without this leading to an extreme rationalist commitment. In the cases of necessary a posteriori statements, the logical principles that they instantiate are of a priori nature, and the information of statements such as "Water is $\mathrm{H}_{2} \mathrm{O}$ " or "Pain is the stimulation of the fiber - $c$ " are knowable only a posteriori. In order to explain this hybridity, how the reductio strategy allows accepting a moderate rationalist approach will be discussed. Bootstrapping will be considered as a paradigmatic case of hybrid epistemic justification. The discussion regarding the a priori - a posteriori nexus tends to establish a hierarchy in which one of the epistemic accesses is privileged, which will be rejected.

\section{Key WORDS}

Necessity, a posteriori, a priori, conceivable, possible

\footnotetext{
"La redacción de este escrito se enmarca en el proyecto FONDECYT Iniciación N 11180422 . Mis sinceros agradecimientos por el respaldo.

** (DD orcid.org/0000-0002-6003-070X Google Scholar
} 


\section{Introducción}

Recientemente, Fischer y Collins (38) han propuesto que la postura kripkeana, concerniente a la discusión necesario - posible, corresponde a un caso de racionalismo modal (modal rationalism), entendiendo éste como un paradigma filosófico que comprende la intuición y los métodos a priori como una vía fiable o confiable de acceso epistémico. Una de las principales consecuencias que se seguiría de ello es que, en casos como $\mathrm{H}_{2} \mathrm{O}-\mathrm{XYZ}$, sostener que $\mathrm{XYZ}$ es concebible conduce a la afirmación de que $\mathrm{H}_{2} \mathrm{O}$ no es una propiedad necesaria de agua. En otras palabras: $\mathrm{Si} \neg \mathrm{F}$ es concebible, entonces $\neg \square \mathrm{F}$.

Esto conduce al descarte de necesidades a posteriori, si su negación es concebible. También conduce a discutir si es plausible afirmar un racionalismo modal kripkeano y si, de ser este el caso, es correcto sostener un nexo concebible - posible cercano a una propuesta bi dimensional epistémica. Fischer y Collins (13) entienden el racionalismo modal como un caso de lo que ellos denominan psicológicamente desinformado (psychologically uninformed), lo que es una falencia desde su propuesta de la filosofía experimental (experimental philosophy). Ocurre lo mismo según los autores con nociones recurrentes en el lenguaje racionalista: intuición, reflexión a priori, inter alia. La alternativa que ellos apoyan, denominada psychologically informed aetiological notion (noción aetiológica psicológicamente informada) tiene entre sus criterios de admisibilidad un sentimiento de ser correcto (feeling of rightness, ídem 15). El objetivo no es el descarte de las intuiciones en la fundamentación filosófica, sino más bien el descarte de intuiciones a priori que no consideran información psicológica relevante (como el criterio de inferencia automática) en la determinación de qué intuiciones son verdaderas, y cuáles deben ser descartadas al carecer de dicha fundamentación. Explícitamente, Fischer y Collins (Ídem 14) alinean el racionalismo modal con el bi dimensionalismo, a través del nexo concebible - posible:

"Modal rationalism suggest that the warrant for the present arguments (N.A: Los casos de lo necesario a posteriori y lo contingente a priori) is provided not...by semantic competence, but through modal insight... in terms of conceivability." (Fischer y Collins 14)

Las consecuencias implausibles que se derivan de un compromiso racionalista con el nexo concebible - posible exigen rechazar esta afirmación. En efecto, recientemente se ha defendido (Miranda, 2017) 
cómo la noción de necesidad débil kripkeana y la dependencia en principios lógicos a priori (identidad, diferencia) permite sostener que los enunciados necesarios a posteriori, si bien corresponden a instancias de esos principios lógicos, no pueden comprenderse como necesarios a priori. La justificación en el caso de lo necesario a posteriori, requiere de información empírica. Explicitado de ese modo (necesidad débil principios lógicos), podría interpretarse que lo necesario a posteriori es un caso híbrido de justificación. Por un lado, los principios lógicos que instancia son de carácter a priori. Por otro, la información de enunciados como "Agua es $\mathrm{H}_{2} \mathrm{O}^{\text {" o }}$ "El dolor es la estimulación de la fibra - $\mathrm{c}^{\prime \prime}$ son cognoscibles sólo a posteriori. Aquí se argumentará que, efectivamente, es un caso de justificación epistémica híbrida, sin que ello conduzca a un compromiso bidimensional epistémico. Para explicar esta hibridez, se considerará el caso del bootstrapping. Previo a ello, se mostrará como la estrategia vía reductio no es suficiente para rechazar la propuesta bidimensional epistémica. La discusión respecto al nexo a priori - a posteriori tiende a establecer una jerarquía, en la que uno de los accesos epistémicos es privilegiado o determina el otro. Siguiendo a Schroeter (2012), esta jerarquización se presenta en el bidimensionalismo semántico:

\begin{abstract}
Influential theorists like David Lewis, Frank Jackson and David Chalmers argue that a generalized 2D semantic framework can be used to isolate an a priori aspect of meaning. Roughly, the idea is that speakers always have a priori access to the truth-conditions associated with their own sentences. On the face of it, this apriority claim seems to conflict with the observation that certain necessary truths, such as 'water $=\mathrm{H}_{2} \mathrm{O}^{\prime}$, can be known only on the basis of empirical inquiry. But proponents of generalized 2D semantics argue that the 2D framework undercuts this objection, by showing how such a posteriori necessities are consistent with a priori access to truth-conditions. (Schroeter 1)
\end{abstract}

El peso de la discusión se encuentra en la noción de condiciones de verdad (truth - conditions). Pues si efectivamente es el caso que los principios lógicos enunciados determinan las condiciones bajo las cuáles sus instancias son verdaderas, entonces parecería correcto sostener que son los principios lógicos en cuestión los que determinan las condiciones de verdad. Diversos autores han enfrentado este problema a partir de las consecuencias semánticas, metafísicas y epistémicas que requiere un compromiso a priorista respecto a los denominados enunciados 
de identidad teórica, incluyendo el análisis epitémico bidimensional propuesto por Chalmers $(2002 ; 2006)$ y las críticas subsecuentes recibidas por Howell, Roca-Royes y Tahko. La idea intuitiva que guía a una postura bidimensional respecto de lo necesario a posteriori es que, en palabras de Nimtz (10): "a sentence is necessary a posteriori if it combines a necessary secondary intension with a contingent primary intension." En particular, esta es la denominada postura epistémica bi-dimensional defendida por Chalmers. Los alcances del nexo concebible - posible en lo necesario a posteriori ponen en cuestión cómo la referencia no puede determinarse por mero análisis conceptual, como es el caso en enunciados como "Soltero es hombre no casado". En esta postura juega un rol relevante la posibilidad de error, carencia de conocimiento (lack of knowledge) o deficiencia de información (under informed). Es una tesis intuitiva que el nexo concebible - posible tiene como principal excepción los casos que respondan a estos criterios.

\section{Rigidez, contexto y externalismo}

Desde los trabajos seminales de Kaplan y Stalnaker, se han discutido los alcances que una propuesta bidimensional tiene en diversos tópicos de la filosofía contemporánea: i) la discusión materialismo - dualismo; ii) la validez del nexo concebible - posible; iii) el rol de lo a priori en la determinación del significado de ciertas expresiones lingüísticas; iv) la validez de lo necesario a posteriori; v) el rol de operadores modales como necesidad, actualidad. Aunque aristas distintas, las problemáticas enunciadas se enfrentan, en primera instancia, con la validez de una postura externalista del significado, la afirmación de que ciertos significados no son determinados a priori. En sus escritos, tanto Kaplan como Stalnaker restringían la aplicación de una propuesta bidimensional a expresiones lingüísticas contextualmente dependientes. La lectura bidimensional, en esos casos, permitía explicar el cambio de referencia en proposiciones que incluyesen indexicales como "Yo" o "Ahora", sin que ello exigiese admitir un cambio del significado del término en cuestión. En ambos casos, la estructura bi-dimensional permitía evaluar el comportamiento de tales expresiones en distintas situaciones, actuales y contrafácticas. A su vez, un caso paradigmático de referencia no dependiente contextualmente es la desarrollada por Kripke, a partir de la noción de rigidez. Términos singulares como nombres propios no varían su referencia en distintas situaciones contrafácticas: son rígidos $\mathrm{y}$, en este sentido, no dependientes del contexto (o mundo posible, o situación contrafáctica). La rigidez que Kaplan sostiene en casos como 
"Yo" es dependiente del contexto, tal que el agente que expresa una oración como "Yo estoy sentado" es designado rígidamente por ese término en toda circunstancia de evaluación (sin considerar el contenido del predicado) en que el agente es el individuo seleccionado por el pronombre.

Distinto es el caso de descripciones definidas como "el mejor jugador de fútbol", que parecen contextualmente dependientes (su referencia varía en distintas situaciones contrafácticas, o al menos así es ampliamente aceptado). En el mundo actual, un enunciado como v.gr. "Messi es el mejor jugador de fútbol" no presenta diferencias en la extensión, pero intuitivamente el que Messi pudiese no satisfacer la descripción "el mejor jugador de fútbol" en un mundo posible o situación contrafáctica distinta de la actual, sugiere no solo un cambio de extensión de la descripción, sino que el significado de ambos términos no es el mismo. Ello tiene la consecuencia modal de que "Messi" refiere rígidamente a Messi, y "el mejor jugador de fútbol" refiere en el mundo actual, pero no en todo mundo posible, a Messi. Es verdadero, en otras palabras, que "Messi es necesariamente Messi", pero no que "Messi es necesariamente el mejor jugador de fútbol". Esta referencia rígida corresponde a una intensión constante, entendiendo por intensión una función que asigna una extensión a cierta expresión lingüística (nombre propio, descripción definida) en cada mundo posible (Schroeter 4). Así ocurre en los casos de semántica unidimensional.

La noción de rigidez sugiere que ciertos términos no parecen estar sujetos a un análisis meramente conceptual en vistas a determinar su referencia y la competencia semántica de un sujeto $S$ respecto a estos términos. Un caso paradigmático y problemático para una postura bidimensional es el de los denominados enunciados necesarios a posteriori, particularmente los enunciados de identidad teórica como "Agua es $\mathrm{H}_{2} \mathrm{O}$ ". ¿Qué aspecto del significado de estos términos es cognoscible a priori? ¿Es su identidad necesaria (si se admite ésta) solo una instanciación del principio de identidad? La idea intuitiva es que esto no es así, que casos como el anterior no son sólo la expresión a priorista de $\mathrm{a}=\mathrm{b}$. Si hay algún aspecto del significado de términos como "agua" u "oro" que sean cognoscibles a priori, parece correcto afirmar que no es lo que permite sostener la identidad a posteriori enunciada previamente. Esto es la postura inicial de un externalismo semántico como el defendido por Kripke y Putnam. Uno de los compromisos centrales de este externalismo es el descarte de que nuestras capacidades epistémicas nos permitan determinar lo que es 
metafísicamente posible. En otras palabras, lo metafísicamente posible es distinto de lo epistémicamente posible. Kripke (124-125) afirma, con base en la evidencia empírica de que "oro" es el elemento cuyo número atómico es 79, que solo es epistémicamente posible (no metafísicamente posible) que oro no sea el elemento con número atómico 79. Es decir, establece los límites del nexo concebible - posible. Cabe destacar, no obstante, que previamente Kripke (123) admite la falibilidad de esta evidencia empírica, lo que complica la defensa de lo necesario a posteriori. Aun cuando la dicotomía epistémicamente posible-metafísicamente posible es la respuesta estándar, no es completamente claro que el could usado en la siguiente cita corresponda al caso de lo epistémicamente posible:
Gold apparently has the atomic number 79. Is it a necessary or contingent property of gold that it has the atomic number 79 ? Certainly we could find out that we were mistaken. The whole theory of protons... on which such views are based, could all turn out to be false. Certainly we didn't know it from time immemorial. So in that sense, gold could turn out not to have atomic number $79^{1}$ (Kripke 123).

Siguiendo a Fischer y Collins, casos de lo concebible no posible corresponden a una ilusión modal (modal illusion). En los casos de lo necesario a posteriori, el criterio de la información empírica es el que permitiría sostener qué casos corresponden a ilusiones modales, y cuáles no. Compárese, por ejemplo, con lo afirmado en Chalmers $(2006,576)$, en que se explicita una lectura de lo metafísicamente posible y no de lo (meramente) epistémicamente posible:

As things has turned out, my terms 'water' and ' $\mathrm{H} 2 \mathrm{O}$ ' have the same extension, and have the same (Kripkean) intension. But there are ways things could have turned out so that the two terms could have a different extension, and a different intension.

Schwartz $(2013,937)$ destaca que esta posibilidad (que no es meramente epistémica) concuerda con la tesis central kripkeana: que un término general de clase natural es no connotativo. De este modo, el significado de "oro" no es being atomic number 79 (Ibid.) Incluso, descripciones de este tipo no están asociadas analíticamente al término general "oro". Respecto

${ }^{1}$ Énfasis de quien escribe. 
a la falibilidad kripkeana enunciada, Schwartz (Ibid.) lo explicita del siguiente modo, en contraste con términos generales connotativos:

We could discover that the chemists are all wrong and that
water is not $\mathrm{H}_{2} \mathrm{O}$.. We could not discover in any sense
that grandmothers are not parents... This is because 'being
a parent' is analytically connected to 'grandmother' but
'being $\mathrm{H}_{2} \mathrm{O}^{\prime}$ is empirically connected with 'water', and
thus 'Water is $\mathrm{H}_{2} \mathrm{O}$ ' is falsifiable like any empirical claim.

Para una postura que exige que el significado de nuestros términos y nuestro acceso epistémico a dicho significado se funde en análisis conceptual a priori, no es admisible una separación entre lo epistémicamente posible y lo metafísicamente posible. El rol dicotómico que cumple un mundo, como contexto de uso (mundo considerado como actual ${ }^{3}$ ) o como circunstancia de evaluación (mundo considerado como contrafáctico ${ }^{4}$ ), adquiere relevancia en casos como los de identidad teórica, pues si se entiende como implícito el operador modal de actualidad, entonces lo que se afirma al sostener "Agua es $\mathrm{H}_{2} \mathrm{O}$ " es "Actualmente, Agua es $\mathrm{H}_{2} \mathrm{O}$ ". Crossley y Humberstone proponen el operador modal complejo de fijación de actualidad en respuesta a casos de referencia invariable, pero dependientes contextualmente (previo a esto, proponen el operador de actualidad como distinto del de necesidad). Si se considera el ejemplo:

“Messi actualmente es el mejor jugador de fútbol.”

Se observa como la denominada intensión diagonal, aquella en que el mundo actual es considerado tanto como contexto de uso y como circunstancia de evaluación (en contextos de uso distintos a M1), no mantiene el valor de verdad del enunciado en los casos que el mundo actual (M1) no es considerado como actual (contexto de uso), sino como contrafáctico (Schroeter 12).

\begin{tabular}{llll}
\hline & M1 & M2 & M3 \\
\hline M1 como actual & V & V & V \\
\hline M2 como actual & F & F & F \\
\hline M3 como actual & F & F & F \\
\hline
\end{tabular}

\footnotetext{
${ }^{2}$ Énfasis de quien escribe. Es importante precisar que el objetivo central del autor es evidenciar una comprensión kripkeana errónea de la postura explicitada por Mill (1843) respecto a los términos generales.

${ }^{3}$ Cf. Chalmers $(2006,577)$.

${ }^{4}$ Ibíd.
} 
Esto abre paso a una comprensión bidimensional de enunciados de identidad teórica, en el que la denominada intensión diagonal permite afirmar la necesidad actualizada de "Agua es $\mathrm{H}_{2} \mathrm{O}$ ", pero impide sostener que esto ocurre en todo mundo posible considerado como circunstancia de evaluación (contrafáctico), no como contexto de uso (actual). Esto es intuitivamente correcto en enunciados que son entendidos como contingentes, como el explicitado "Messi es el mejor jugador de fútbol", pero esas intuiciones no parecen exigir el descarte de:

i) enunciados necesarios no fijados actualmente

ii) enunciados necesarios no analíticos, a posteriori.

La exigencia de que sólo enunciados necesarios a priori tengan en cada caso de la intensión diagonal el valor de verdad verdadero $(\mathrm{V})$, coincide con la comprensión bidimensional de que lo necesario a posteriori tiene, sin excepción, un aspecto del significado de carácter contingente:

Using 2D modal logic, it's easy to construct necessary a posteriori truth. The semantic rules governing the modal operator ' $A$ ' (N.A: Actualidad) guarantee that every claim of the form AS will be either necessarily true or necessarily false in the sense of ' $\square$ '. But when the embedded sentence $S$ is an ordinary empirical truth like 'Obama won', AS will be knowable only a posteriori: so AS will be a necessary a posteriori truth. (Schroeter 2012, 13)

La distinción central entre un esquema uni dimensional en contraste con uno bi dimensional es el modo cómo se determina la asignación de una extensión E como el valor semántico de cierto término T. En un esquema uni dimensional, se considera sólo el mundo actual. En un esquema bi dimensional, se considera el mundo actual y un mundo posible. Esta comprensión exige, entre otros aspectos problemáticos en relación con los términos generales, la determinación de una cierta clase $\mathrm{C}$ a partir de la extensión de dicha clase $C$. Además, descarta la legitimidad de enunciados necesarios que dependan en la determinación de sus condiciones de verdad de estados de cosas del mundo actual. Al comprender la actualidad como un operador modal contextualmente dependiente (y por lo tanto variable de acuerdo con qué mundo o situación se entiende como actual), es una consecuencia esperable el rechazo de lo necesario a posteriori como caso paradigmático de una necesidad contextualmente dependiente. Simplemente, no es metafísicamente necesario que "Agua es $\mathrm{H}_{2} \mathrm{O}^{\prime \prime}$, pues dependiendo de la situación contrafáctica que cumpla el 
rol de contexto de uso (en contraste con circunstancia de evaluación), puede resultar falso que, en el mundo considerado como actual, "Agua es $\mathrm{H}_{2} \mathrm{O}^{\prime \prime}$. Las intuiciones respecto a que esa conclusión sería incorrecta, dependen de considerar el mundo actual como contexto de uso en toda situación contrafáctica. En otras palabras, de fijar la referencia. La intuición uni dimensional en este punto es que "Agua", "Oro" y términos similares, se comportan como términos rígidos sin variación de extensión, a diferencia del caso dependiente contextualmente de "Yo". "Agua" sería un término contextualmente invariante (context invariant). Las intuiciones concernientes a que éste no sería el caso, sitúan los términos de clase a la par de los indexicales. Por ejemplo, Jackson (1998a; 1998b), afirma que "Agua" puede ser entendido como una expresión indexical implícita (implicit indexical expression). A su vez, Chalmers $(2006,575)$ explícitamente compara el par de términos "Yo" - "David Chalmers" con "Agua" " $\mathrm{H}_{2} \mathrm{O}$ ", al afirmar que la intensión (entendida como la función desde los mundos posibles a las extensiones) de "Agua" es " $\mathrm{H}_{2} \mathrm{O}^{\prime}$. Esto descarta, contra lo defendido en Miranda (2012), que "Agua" se entienda como un término rígido de jure, pues ello exige que el contenido semántico sea el referente, en este caso la clase Agua, y no " $\mathrm{H} 2 \mathrm{O}$ ". En el caso de un término general, Chalmers comprende la extensión del término general como: "... the class of individuals that fall under the term... the extension of 'cat' is the class of cats." (Ibid.). Esto evidencia el descarte de la distinción designación - denotación, el sostener que un término de clase $C$ refiera abstractamente la clase $\mathrm{C}$ (y no las instancias de la clase). Más importante, Chalmers atribuye a Kripke la afirmación de que " $\mathrm{H}_{2} \mathrm{O}$ " es la intensión de "agua", lo que no es correcto si "agua" corresponde a un término rígido de jure. Del mismo modo, Chalmers ubica los enunciados de identidad teórica como un caso de enunciado contingente, similar a "Messi es el mejor jugador del mundo":

...there is a sense in which for a term like 'water', the
term's extension and its Kripkean intension depend on
the character of our world. Given that this world is actual,
it turns out that 'water' refers to $\mathrm{H}_{2} \mathrm{O}$, and its Kripkean
intension picks out $\mathrm{H}_{2} \mathrm{O}$ in all possible worlds. But if another
world had been actual (e.g. Putnam's Twin Earth world in
which $\mathrm{XYZ}$ is the clear liquid in the oceans), 'water' might
have referred to something quite different (e.g. XYZ), and
it might have had an entirely different Kripkean intension
(e.g. one that picks out XYZ in all worlds) ${ }^{5}$ (Chalmers 576).

${ }^{5}$ Respecto a la relevancia del experimento mental de la tierra gemela en la discusión concebible - posible, ver Miranda (2017). 
Las intuiciones respecto al nexo concebible - posible que se observan en afirmaciones de este tipo son cuestionables, y han sido ampliamente discutidas durante la última década. $\mathrm{Si}$, de alguna manera, esas intuiciones son incorrectas, o si conducen a tesis que requieren de evidencia empírica para ser sostenidas (por ejemplo, qué criterios descriptivistas debería cumplir el concepto "agua", no por mera vía condicional a priori sino por información empírica), la postura bidimensional epistémica se encontraría ante el problema de que el acceso a priorista no basta para justificar de qué modo XYZ no existe, aun si es concebible. En última instancia, se trata de cómo lo concebible no es siempre una guía fiable hacia lo metafísicamente posible. Para Chalmers, no es correcto sostener que $\mathrm{XYZ}$ no existe. Debe considerarse que, desde una postura bi - dimensional, casos en los que agua no es $\mathrm{H}_{2} \mathrm{O}$ son concebibles, epistémicamente posibles, y metafísicamente posibles. Lo último, debido a que la dicotomía contex to de uso - circunstancia de evaluación permite afirmar posibilidad no meramente epistémica, sino que metafísica. La propuesta bidimensional supone que la determinación de la referencia es de carácter internalista, contra el externalismo semántico. Es eso lo que se discutirá en la siguiente sección.

\section{Determinación referencial e internalismo: los alcances del nexo concebible - posible}

On the post - Kripkean picture, even if it is not necessary that not $-\mathrm{P}$, it may still be a priori that not - $\mathrm{P}$ (contingent a priori); and even if it is not a priori that not - $\mathrm{P}$, it may still be necessary that not - $\mathrm{P}$ (necessary a posteriori)... it may be possible that $\mathrm{P}$ but not Conceivable that $\mathrm{P}$, or Conceivable that $\mathrm{P}$ but not possible that $\mathrm{P}$... the necessary a posteriori seems to guarantee that there will be cases of Conceivability without possibility (Gendler \& Hawthorne 33).

La determinación de la referencia (reference determination) es, probablemente, el problema más urgente que una postura bidimensional intenta solucionar vía a priori. El objetivo declarado por Chalmers (2004; 2006a) es mantener el triángulo dorado (golden triangle) entre significado (meaning), a prioridad (apriority) y posibilidad (possibility). Esto conduce a la tesis intuitiva de que lo que es epistémicamente concebible, es posible. $Y$ esta tesis sugiere que lo que no es epistémicamente concebible, no es posible. El nexo: 
$\mathrm{C} / \mathrm{P}$

conduce a

$\neg \mathrm{C} / \neg \mathrm{P}$.

Ahora bien, esto exige un acceso internalista a las condiciones que permiten determinar la referencia. En múltiples casos, incluso los descriptivistas considerados por Chalmers, el acceso a esas condiciones o criterios de determinación de la referencia es externo, empírico. Al afirmar que el significado de términos como "Héspero" o "Fósforo" no es el referente, sino cierto contenido descriptivo (o connotativo no descriptivo $\left.{ }^{6}\right)$, se establece una vía apriorista para determinar si tales términos son co-referenciales. Chalmers (2002) centra su análisis precisamente en la pregunta de si lo concebible implica lo posible (Does conceivability entail possibility?). La idea intuitiva, y que no se intenta rechazar, es la que explicita Chalmers $(2002,146)$ : “...there is at least some plausibility in the idea that conceivability can act as a guide to metaphysical possibility."

El punto central que refleja esta cita es la distinción entre que concebibilidad implique posibilidad y concebilidad actúe como guía hacia la posibilidad metafísica

Chalmers comprende la posibilidad metafísica como más amplia que la posibilidad física o natural. El ejemplo que da es: “...it seems conceivable that an object could travel faster than one billion meters per second. This hypothesis is physically and naturally impossible, because it contradicts the laws of physics and the laws of nature." (Ibíd.)

El criterio para descartar esta posibilidad física es que implica contradicción, o que contradice las leyes actuales de la naturaleza. No obstante, se asume metafísicamente posible un caso como éste, pues se asume que la posibilidad metafísica sí considera como posibles casos de este tipo, ante eventuales leyes de la naturaleza distintas de la actual. Volviendo a la dicotomía enunciada actuar como guía - implicación, la primera no exige la segunda. Actuar como guía admite casos de lo concebible no posible, excepciones a $\mathrm{C} / \mathrm{P}$, tal que $\mathrm{C} / \neg \mathrm{P}$

${ }^{6}$ Kaplan (1989). 
Precisamente estos casos son los que consideran los criterios de carencia de conocimiento (lack of knowledge) o deficiencia de información (under informed). Admitir casos de este tipo exige el rechazo del triángulo dorado:

significado (meaning) - a prioridad (apriority) - posibilidad (possibility).

Chalmers (Ibid.) propone, análogo al caso de actuar como guía, el nexo concebible - posible entendido como reflejo de: “...in this case (N.A: el objeto que viaja más rápido que un billón de metros por segundo) although conceivability does not mirror natural possibility, it may well mirror metaphysical possibility."

Ya no afirma ni implicación, ni ser guía, sino que un reflejo, un espejo de la posibilidad metafísica. Ser reflejo de parece suponer al menos implicación. Esto conduce a la tesis implausible de que:

\section{T1: Posibilidad exige Concebibilidad.}

Que debe ser concebido algo como posible, para que se pueda afirmar su posibilidad, el denominado bi dimensionalismo epistémico. Chalmers (Ibid.) explicita este punto al afirmar:

"It is...widely believed that empirical identities provide counterexamples: on this view, it is conceivable but not possible that Hesperus is not Phosphorus, and that water is not $\mathrm{H}^{2} \mathrm{O}^{7 \prime}$

Chalmers propone tres dicotomías de concebibilidad:

Prima facie - ideal

Primaria - secundaria

Positiva - negativa

Y sostiene que hay al menos un caso (ideal) en el que la noción de concebibilidad resulta incólume de críticas como la de "Agua es $\mathrm{H} 2 \mathrm{O}$ ". Si se considera lo afirmado en Chalmers $(2006,576)$, no se trata de lo epistémicamente posible, pues esa alternativa ya está cubierta por Kripke, y es discutida ampliamente en Soames (2011). Pese a ello, lo epistémicamente posible es considerado como un punto de inicio de una estrategia bi-dimensional: "...it seems to be at least epistemically

${ }^{7}$ Énfasis de quien escribe. 
possible... that these terms (N.A: 'water' $\mathrm{y}$ ' $\mathrm{H} 2 \mathrm{O}$ ' inter alia) might fail to co - refer... cognitive differences between the terms is connected in some fashion to the existence of these possibilities" (Ibíd.).

La diferencia cognitiva entre términos co-referenciales ha sido discutida en XXX (2013b), y para efectos de este escrito es relevante enfatizar de qué modo esta diferencia conduciría o exigiría una lectura bi-dimensional. La tesis implícita es que lo epistémicamente posible es una guía a lo metafísicamente posible, y que las diferencias cognitivas estarían fundadas en esta evaluación modal bidimensional en la que no es sólo concebible, sino metafísicamente posible, que agua no es $\mathrm{H}_{2} \mathrm{O}$.

\section{El descarte de la vía reductio como solución al nexo concebible - posible}

Si una reducción al absurdo (reductio ad absurdum $\left.{ }^{8}\right)$ fuese viable en vistas a descartar la posibilidad de una lectura metafísica bidimensional, una de las consecuencias directas de esta reductio sería la separación del nexo concebible - posible. Por más intuitivo que sea este nexo, lo cierto es que afirmarlo conduce a compromisos a prioristicos y el descarte de necesidad a posteriori, strictu sensu. En un escrito reciente, Howell (2008) explora esta línea de defensa de lo necesario a posteriori vía ignorancia o carencia de conocimiento. El criterio de ignorancia es importante, pues la defensa de la necesidad de la identidad conduce a que, si un enunciado de identidad teórica es rechazado como necesario por el bi dimensionalismo, el único modo en que se puede fundamentar este rechazo es a partir del desconocimiento de esta identidad. La otra alternativa, que parece descartada, es una defensa de identidades contingentes. Sostiene Howell (Idem., 349): "If we are ignorant of the identity of water and $\mathrm{H}_{2} \mathrm{O}$ (or ignorant of the necessity of identities) then we can perhaps conceive of a world where water is something else, but this is not indication of its possibility."

Lo primero que podría criticarse al supuesto de ignorancia enunciado, es que no correspondería a la situación epistémica defendida por Chalmers (2002; 2006), pues en tales casos no se trataría de que exista una carencia de conocimiento de que es verdadero en el mundo actual que "Agua es $\mathrm{H}_{2} \mathrm{O}$ ". En el caso de un bidimensionalismo epistémico,

\footnotetext{
${ }^{8}$ Siguiendo a Rescher (2002), la reductio puede presentarse de tres modos: i) una auto contradicción (ad absurdum); ii) una falsedad (ad falsum o ad impossible); y iii) una anomalía o implausibilidad (ad ridiculum o ad incommodum)
} 
se asume que las condiciones epistémicas ideales descartan carencia o información limitada. La necesidad de la identidad sería, en ese caso, la tesis rechazada por el bi dimensionalismo. De cualquier modo, se trataría de algún tipo de carencia de información. Esto no es extraño, pues la única intensión diagonal aceptada como verdadera en toda situación contrafáctica es la de enunciados analíticos como "todo soltero es no casado". Y son casos como estos los que se aceptan como necesarios debido a su no dependencia empírica. Otra tesis central es, por tanto, que no hay necesidad a posteriori a la Kripke: la intensión primaria es contingente, y la secundaria necesaria. Gendler \& Hawthorne (2002, 32) afirman que casos como "Héspero es Fósforo" y "Agua es $\mathrm{H}_{2} \mathrm{O}$ " corresponden a casos de necesidad a posteriori porque:

They are necessary because the rigid designators on each side of the copula co - refer in the actual world, and hence in all possible worlds; but they are knowable only a posteriori because the co - reference of the terms is itself contingent. ${ }^{9}$

No es por este motivo que Kripke sostiene el acceso epistémico a posteriori de verdades necesarias. De hecho, Kripke previene explícitamente esta interpretación de la co - referencialidad, al distinguir entre

i) que dos términos designen rígidamente y ii) que dos términos puedan variar su referencia y que, por lo tanto, no designen rígidamente un objeto o en toda situación contrafáctica (rigidez obstinada).

La afirmación de Gendler y Hawthorne se comprende mejor si se tiene en vistas el modo en que ellos entienden la noción de proposición necesaria: "Whether a proposition is necessary or contingent... it turns on whether its truth is independent of what the world is like ${ }^{10 "}$ (idem., 31). Esto descarta la información empírica como criterio que determine la necesidad de una proposición P. Esta comprensión de lo necesario $a$ posteriori permite explicar cómo "Agua no es $\mathrm{H}_{2} \mathrm{O}$ " tiene una intensión primaria que es verdadera en algún mundo posible, aunque su intensión secundaria sea falsa (o verdadera) en cada mundo posible. En palabras de Howell (ídem., 349 - 350):

\footnotetext{
${ }^{9}$ Énfasis de quien escribe.

${ }^{10}$ Énfasis de quien escribe.
} 
The necessary a posteriori, says the two dimensionalist, occurs at the level of statements. 'Water is $\mathrm{H}_{2} \mathrm{O}^{\prime}$ ' is an example of the necessary a posteriori because it is associated with two different propositions. In Chalmers terminology, it has a primary intension and a secondary intension... Statements that are necessary a posteriori, like 'water is $\mathrm{H}_{2} \mathrm{O}^{\prime}$... have a contingent primary intension but a necessary secondary intension

Esto responde a los intereses en vistas a mantener el nexo concebible posible y mantener el triángulo dorado a priori - significado - necesidad (posibilidad). Si es concebible que agua es XYZ, entonces es posible. La estrategia bi dimensionalista epistémica asume que la intensión primaria, entendida como el modo en que un sujeto $S$ concibe el mundo $\mathrm{M}$, exige que $\mathrm{M}$ sea metafísicamente posible, no meramente concebido. $\mathrm{Si}$ esta estrategia es generalizada, conduce a la afirmación expresada por Howell (Idem. 351): "Any view that combines two dimensionalism with the view that there are always metaphysically possible worlds that make coherent primary intensions true is what I call metaphysical two - dimensionalism."

Es esto lo que se afirma en casos $\mathrm{como}_{2} \mathrm{O}-\mathrm{XYZ}$ : no mera concebibilidad, sino que posibilidad metafísica. Aquí entra la estrategia vía reductio. El esquema central de una reductio es el siguiente:
a) Se asume $p$
b) Se argumenta cómo, asumiendo $p$, se sigue $\neg$ p.
c) Se sostiene p \& $\neg$ p: contradicción.

Howell observa cómo la postura bidimensional epistémica requiere que la concebibilidad de la intensión primaria asegure su posibilidad. Pero, si se halla un caso de intensión primaria o secundaria concebible aunque metafísicamente imposible, el criterio de coherencia interna sería débil para establecer la posibilidad metafísica. En otras palabras, apelar a escenarios concebibles, pero no posibles o, como se enunció anteriormente (p. 9), el re-establecimiento de:

$\mathrm{C} / \neg \mathrm{P}$

Chalmers $(2002,189)$ sugiere que casos de lo concebible no posible corresponden a casos de lo concebible negativo prima facie. Esta estrategia requiere asumir acceso epistémico a lo no posible vía lo concebible, 
estrategia que depende de carencia de conocimiento (lack of knowledge) o, más débil, de acceso epistémico a un ítem de lo concebible no posible ${ }^{11}$.

El escenario en cuestión es el siguiente, denominado Strong Neccessity $(\mathrm{SN}):$ " $(\mathrm{SN})$ The space of metaphysically possible worlds is more limited than the space of conceivable worlds" (idem. 152).

El supuesto es que, efectivamente, es concebible un escenario de este tipo ¿Por qué? Fundamentalmente por la coherencia interna. Podría argumentarse, contra este criterio, que su contradictoria sería igualmente concebible, en vistas a su coherencia interna:

a) The space of metaphysically possible worlds isn't more limited than the space of conceivable worlds.

Antes de responder a esta argumentación, considérese lo que pretende en primer lugar SN, a saber: mostrar cómo se puede enunciar un caso de lo concebible no posible, a partir de una postura bidimensional epistémica. En efecto, si SN es verdadero, lo concebible no implica lo posible, es decir:

b) Hay al menos un caso de un mundo $M$ concebible que no es metafísicamente posible.

Esto sería una consecuencia directa de limitar lo metafísicamente posible, pero es una limitación que, en tanto concebible, no es admisible de ser cuestionada por el bi dimensionalismo epistémico. En otras palabras, para rechazar SN, la postura bi dimensional epistémica debería descartar que SN es concebible, y esto no parece plausible. Basta considerar que, si ese fuese el caso, su negación tampoco debiese ser concebible. En última instancia, la pregunta central es que casos (distintos de los de incoherencia interna) no son concebibles. Las limitaciones epistémicas ofrecen una vía de solución por defecto, en tanto casos no concebidos son (actualmente), por defecto, no concebibles. Si bien es cierto Howell otorga a este escenario el carácter de una verdad necesaria, aplicable a todo mundo posible, es esta consecuencia la que conduce al cuestionamiento de si su contradictoria no es del mismo modo concebible. En la nota 9, Howell explicita porque SN debiese ser entendido como una verdad necesaria, fundado en que el bi dimensionalismo epistémico admite S5 y, en S5

${ }^{11}$ Howell $(2008,354)$ discute este caso a partir de la propuesta metamodal desarrollada por Yablo (1999), y rechazada por Chalmers (2002). 
c) Si SN es verdadero en un mundo posible $\mathrm{Mp}$, SN es posible desde el mundo actual Ma.

Ya que SN es acerca de todo el espacio lógico (The space of metaphysically possible worlds. Howell Idem., 352), se sigue que

d) Si SN es verdadero, es necesariamente verdadero.

En la reductio propuesta, la omisión de la concebibilidad de la contradictoria de SN adquiere importancia, si su rol impide la afirmación de esta verdad necesaria supuesta. Es decir, si es concebible tanto

e) SN: The space of metaphysically possible worlds is more limited than the space of conceivable worlds.

Como

f) $\neg \mathrm{SN}$ : The space of metaphysically possible worlds isn't more limited than the space of conceivable worlds.

o

$\left.f^{\prime}\right) \neg$ (The space of metaphysically possible worlds is more limited than the space of conceivable worlds.)

Entonces podría afirmarse que la reductio fracasa en al menos un punto, a saber: el descarte de que el bi dimensionalismo epistémico no tenga una defensa contra la reductio vía negación de $\mathrm{SN}$, aun cuando SN sea concebible. Esto pondría en evidencia que tanto

g) $\mathrm{SN}$ como $\neg \mathrm{SN}$ tienen una intensión primaria contingente.

Pues, si no lo fuese, coincidiría con los casos de intensiones diagonales analíticamente necesarias. Y, en ese caso, no sería concebible tanto SN como $\neg \mathrm{SN}$, pues en ese caso la negación de SN no sería concebible, como no es concebible la falsedad de "todo soltero es no casado".

En otras palabras:

h) SN no corresponde a un caso de necesidad fuerte, pues su intensión primaria es contingente.

El problema es que la intensión primaria no puede comprenderse como contingente, pues la intensión primaria en casos como $\mathrm{SN}$ es determinada a priori. Howell afirma esta aprioridad de SN, debido a la imposibilidad de contrastación empírica de un enunciado acerca de todo el espacio lógico: "If it was a posteriori, one wonders how it could 
be empirically established" (Ibid.) Si un punto parece estar fuera de discusión desde Chalmers (2002; 2006) es que,

i) Si la intensión primaria se entiende como contingente, no puede ser a priori.

Esto responde al desiderata inicial del triángulo dorado, pues el objetivo es que el aspecto del significado inmune a revisión empírica a posteriori, se encuentra en la intensión primaria. De modo indirecto, Howell (Ibid.) sostiene lo mismo al exigir que, para la postura bidimensional, $\mathrm{SN}$ sea considerado como un enunciado a priori falso, pues al ser sobre todo el espacio lógico no es viable su contrastación empírica. La consecuencia positiva contra un bi dimensionalismo epistémico es que, considerando la dicotomía concebible ideal - concebible prima facie.

No puede afirmarse que SN es meramente prima facie concebible, pues ello exigiría sostener un acceso epistémico que evite cierta carencia de conocimiento en relación con SN. Es decir, el descarte de que SN sea idealmente concebible. Al respecto, sostiene Howell (354): “The person who conceives of the possibility of SN might simply fail to be conceiving ideally... Chalmers will presumably... simply deny that SN is ideally conceivable, even if it is prima facie conceivable."

El cuestionamiento de la circularidad de lo que es idealmente concebible (concebible si y sólo si implica posibilidad), muestra de qué modo no es una respuesta satisfactoria a $\mathrm{SN}$ :

j) En tanto SN y su negación son concebibles, no hay un criterio empírico que permite determinar cuál de los dos corresponde a una ilusión modal ${ }^{12}$.

La vía reductio no es una solución al nexo concebible - posible, pues los supuestos que asume exigen un compromiso con la concebibilidad de la negación de SN. En la siguiente sección, se discutirá una alternativa de justificación híbrida en vistas a evitar el compromiso racionalista con lo concebible: el denominado bootstrapping.

${ }^{12}$ Sobre este punto, ver Miranda (2017). 


\section{Bootstrapping: una estrategia híbrida de justificación}

...there is a more fundamental problem underlying the a priori/a posteriori distinction - one that undermines even the obvious solution of restricting a posteriori knowledge to perceptual information and a priori knowledge to non - perceptual information. The problem we have in mind is the following: no matter how strictly one might restrict knowledge to experience...I $\mathrm{t}$ seems that there are always also some a priori elements present (Tahko 153).

Una solución a esta cuestión, que evita las consecuencias metafísicas de un compromiso concebible - posible, pero que al mismo tiempo rescata la intuición de que en ciertos casos este nexo es confiable, lo constituye la propuesta del bootstrapping (empuje, arranque). Dicha noción surge de la discusión epistémica respecto a qué justificación tiene un sujeto $S$ para creer que algún enunciado $\mathrm{E}$ es verdadero (o falso). Si es el caso que dicha justificación en creer P supone la confiabilidad en ciertos ítems de conocimiento que suponen $P$, entonces se presenta el problema escéptico de qué justifica la creencia (o conocimiento) en $\mathrm{P}$. El caso paradigmático es el de la percepción, y se ha discutido recientemente en otro escrito ${ }^{13}$. Lo que interesa ahora es rescatar la arista positiva del bootstrapping en la discusión a priori - a posteriori.

Recientemente, Tahko (2011) ha propuesto que la jerarquización a priori a posteriori, supuesta en casos como el bidimensionalismo epistémico es la causa del problema: es un error epistémico establecer esta jerarquía, pues lo que ocurre en los casos de justificación es una interdependencia de aspectos a priori y a posteriori. En la sección anterior, se discutió cómo la vía reductio no consigue refutar el bi dimensionalismo a través de SN. Se considera que la principal razón de este fracaso es la suposición de la jerarquía a priori - a posteriori. En efecto, es esta jerarquización la que exige que, si SN es concebible, también lo es $\neg \mathrm{SN}$. En los casos paradigmáticos de enunciados necesarios a posteriori, la pregunta central es cómo un estado actual del mundo puede justificar un enunciado necesario. Siendo en estos casos el problema central el nexo a priori - a posteriori, una alternativa que rescate la relevancia de ambos ítems en el acceso epistémico a ciertos enunciados tiene una ventaja explicativa. Del mismo modo, en discusiones contemporáneas respecto a objetos meramente concebibles, es la ausencia del ítem a posteriori el que conduce a un

$\overline{{ }^{13} \text { Cf. Miranda (2013). }}$ 
rechazo de, por ejemplo, la posible existencia de zombies ¿Por qué parece erróneo sostener que un zombie es posible, aun siendo concebible? Roca - Royes y Polcyn intentan responder esto. La noción de bootstrapping es comprendida en este caso como una relación entre lo a priori y lo a posteriori. Tahko (152) lo explicita del siguiente modo: “... a priori and a posteriori knowledge are in constant bootstrapping relationship... we introduce an a priori proposition, which we then attempt to verify by a posteriori means; this is the core of the bootstrapping relationship."

El criterio de verificación, luego complementado con el de falsificación, conduce a Tahko a sostener la falibilidad del conocimiento que se obtenga de esta relación. Falibilidad que, no obstante, no descarta confiabilidad en el acceso a dicho conocimiento, sino que más bien permitiría aceptar el proceso epistémico como acumulativo (Ibid.). El caso que mejor ejemplifica este nexo a priori - a posteriori es el de los principios lógicos, en particular el principio de no contradicción. Al no encontrar un contraejemplo a dicho principio en el ámbito empírico, su validez no es meramente conceptual. Esto lo explicita Roca - Royes $(2011,24)$ en su comprensión de lo concebible á la Yablo:

\begin{abstract}
The Greeks, according to Yablo, could conceive $\mathrm{Y}$ of water being other than $\mathrm{H}_{2} \mathrm{O}$ (due to their lack of chemical concepts and their lack of knowledge that water is $\mathrm{H} 2 \mathrm{O}$ ). However, water is not $\mathrm{H}_{2} \mathrm{O}$ is not conceivable $\mathrm{Y}$ for a contemporary subject who knows that water is $\mathrm{H}_{2} \mathrm{O}$ (the idea is that that would require conceiving of a contradiction: $\mathrm{H}_{2} \mathrm{O}$ not being $\left.\mathrm{H}_{2} \mathrm{O}\right)$.
\end{abstract}

Esto es distinto a lo propuesto por el denominado plan Canberra explicitado por Fischer y Collins (9), cuyos principales exponentes son Lewis (1994) y Jackson (1998). En ambos casos, la reflexión a priori (la denominada armchair reflection) sistematiza las intuiciones que un sujeto $\mathrm{S}$ tiene respecto a $\mathrm{F}$, y ulteriormente estas intuiciones son contrastadas empíricamente. El bootstrapping, por otra parte, no supone que el acceso sea desde lo a priori a lo a posteriori, sino que permite una interrelación flexible en ambas direcciones. No obstante, las afirmaciones modales concernientes a la posibilidad o la necesidad, son consideradas por Tahko como a priori. El que el principio de contradicción se cumpla en la realidad muestra una vía de incidencia de lo necesario y lo posible en lo empírico, y las intuiciones son confirmadas de este modo vía $a$ posteriori. La intuición de que si algo es idéntico a sí mismo, entonces 
es necesariamente idéntico a sí mismo, guían las afirmaciones respecto a la imposibilidad (y no solo la falsedad) de que "Agua no es $\mathrm{H}_{2} \mathrm{O}^{\text {" }}$ sea verdadera. Esto es sólo un caso paradigmático de enunciado de identidad teórica, y permite mostrar en qué sentido las intuiciones modales kripkeanas no son racionalistas, al menos no en el sentido de que estas intuiciones no requieran de información empírica para que sean entendidas como verdaderas. Desde este punto de vista, las intuiciones son:
i) Falibles
ii) Confiables
iii) ${ }^{y}$ Verificables

Una pregunta que sólo será enunciada en este escrito es: ¿Cómo puede lo necesario a posteriori responder al criterio de falibilidad exigido por el avance de la información empírica? Tahko (2011, 159 - 160) otorga relevancia en este punto a una estrategia epistémica que no explica en detalle: el backtracking (volver atrás). Aceptando la falibilidad de la información empírica, el backtracking constituye el retorno a un punto previo del árbol (tree) del conocimiento, que prevenga su terminación en un callejón sin salida (dead - end). En algún sentido, el backtracking es parte de la relación de bootstrapping, pues el nexo a priori - a posteriori considera las posibilidades de error o carencias de información que puedan exigir el backtracking. Lo metafísicamente necesario no se circunscribe a las intuiciones o la reflexión racional a priori. Lo concebible qua concebible no es, de este modo, una guía segura de fundamentación modal, especialmente en el caso de lo necesario a posteriori. Esto es el centro del externalismo defendido por Kripke , Putnam y Burge (1979, 1986), y de la distinción kripkeana entre lo epistémicamente posible (lo concebible) y lo metafísicamente posible. El bootstrapping no supone un rol infalible de las intuiciones, como si lo hace el racionalismo modal que Fischer y Collins atribuyen a Kripke (1980):

\footnotetext{
Whereas in the past intuitions were elicited to elucidate the content of concepts, they were now probed to determinate metaphysical necessities. The philosopher, according to such 'modal rationalism', does not so much as analyse concepts independently of the way the world is, but uses intuitions to gauge and determinate modal claims about how the world must be.
} 
Una razón por la que el denominado racionalismo modal ha sido comprendido como plausible es que, al parecer, enunciados modales no son strictu sensu empíricos, a posteriori. Un caso cotidiano es el explicitado por Roca - Royes $(2011,23)$ :

I know that the pen on my desk could be on top of the pile of papers, rather than next to it. If someone challenged this piece of knowledge, I would move the pen and place it on top of the pile of papers.

En estos casos, si bien empíricamente verificables, se explicita cómo los enunciados modales no son a posteriori. Tahko tiene presente ejemplos de este tipo al cuestionar la distinción entre a priori - a posteriori discutida por BonJour (1998) a partir de los criterios de:

a) qué se entiende por experiencia y

b) si lo a priori es independiente de la experiencia.

La arbitrariedad de esta distinción es evidente cuando se entiende el acceso epistémico a verdades paradigmáticamente entendidas como $a$ priori, vía a posteriori $(v \cdot g r .2+2=4)$. Igualmente arbitrario sería sostener que, por este motivo, todo conocimiento es a posteriori. Es el intento de evitar esta arbitrariedad la que impulsa la propuesta del bootstrapping. Un aspecto interesante, quasi racionalista de esta relación, es que según Tahko $(2011,155)$ el rol de lo a posteriori es determinar cómo son las cosas en el mundo actual. En efecto, en un escrito previo a la propuesta del bootstrapping como una vía de solución a la dicotomía a priori - a posteriori, Tahko (2009) sostiene explícitamente que el contenido modal de los enunciados necesarios a posteriori no es a posteriori:

... What is the modal content of a posteriori necessities based on; in virtue of what are they necessary? At least in most cases, the modal content seems to come from the a priori part. To take one of the usual examples, consider the identity statement 'Hesperus is Phosphorus'. We can analyse it as follows. We know a posteriori that Hesperus is in fact Phosphorus, that is, we know that the identity statement 'Hesperus is Phosphorus' is true. Further, we know a priori that if Hesperus is Phosphorus, then it is necessary that Hesperus is Phosphorus. Clearly, Hesperus is necessarily Phosphorus in virtue of the a priori part. (Tahko 2009, 344). 
En este punto, no se aleja de lo afirmado por McLeod (2009), lo que se puede denominar un tipo de racionalismo modal no a priorístico. El nexo entre las observaciones empíricas y las deducciones que se derivan de estas es, desde este análisis, una de las vías de lo necesario a posteriori. Ocurre así con el caso citado de co-referencialidad rígida Héspero - Fósforo, y en los casos de enunciados de identidad teórica. La otra vía es la búsqueda de hipótesis no contrastadas (previamente) empíricamente ${ }^{14}$. Es en casos de este tipo en que lo concebible adquiere relevancia. Ocurre así en el denominado gravitational redshift (cambio gravitacional al rojo), en el que Tahko observa un proceso de bootstrapping desde la teoría corpuscular newtoniana (falsificada vía a posteriori) hasta la teoría de relatividad especial einsteniana, y la confirmación desarrollada por el experimento Pound - Rebka en 1959 (verificación vía a posteriori). Hay que destacar que casos de este tipo descansan en la no acomodación de la información empírica (Tahko 2011, 157) en ambas teorías, pero los casos de inconmensurabilidad complican la defensa del bootstrapping. La propuesta central supone que los casos de inconmensurabilidad no son frecuentes.

La terminología que utiliza Tahko (Idem., 158) para referirse a lo concebible es la noción de meramente posible (merely possible). Esta comprensión explica cómo la relación del bootstrapping entre lo a priori y lo a posteriori asume el mundo actual como criterio de posibilidad: la relación de bootstrapping corresponde a un caso de actualismo:

We can imagine the bootstrapping relationship as a tree model: we start with a single trunk that represents the established a posteriori basis... The branches of the tree represent lines of a priori reasoning, a priori propositions. But not all of these a priori propositions are true, some are merely possible. ${ }^{15}$

Ulteriormente, Tahko (ídem., 161) establece una relevante distinción entre lo a priori y lo concebible:

...we also wish to strongly resist reducing a priori reasoning
to conceivability or conceptual analysis... everything from
philosophical zombies to Disney fairy tales is conceivable:
indeed, conceivability appears to be entirely unrestricted.

\footnotetext{
${ }^{14}$ Ocurre así en los casos de aplicación modal del diario vivir, como es el caso del problema del automóvil y sus posibles razones. Cf. Tahko $(2011,156)$.

${ }^{15}$ Énfasis de quien escribe.
} 
But the tree of knowledge cannot be unrestricted, we have to be able to rule out metaphysically impossible scenarios that might nevertheless be conceivable ${ }^{16}$

La vía epistémica a priori es la que, de modo confiable, permite descartar ciertos escenarios concebibles, pero metafísicamente imposibles. Y la relación de bootstrapping es la que permite determinar qué escenarios son metafísicamente imposibles, y cuáles no, a partir de la información $a$ posteriori disponible. Como sostendrá en un escrito reciente Tahko (2015, 795), la intuición no es criterio epistémico suficiente para fundamentar, entre otros aspectos, la validez de principios lógicos a priori.

\section{Conclusión}

El nexo concebible - posible supone una jerarquía epistémica a priori - a posteriori. El presente escrito analizó las consecuencias implausibles de este supuesto, particularmente el compromiso con objetos meramente concebibles. La estrategia vía reductio no soluciona el desafío bi dimensionalista epistémico, pues asume que $\neg \mathrm{SN}$ no es concebible. La propuesta epistémica del bootstrapping supone un avance en la discusión, al considerar la interrelación a priori - a posteriori de modo no jerárquico. La carencia de conocimiento empírico continúa siendo un criterio relevante en la determinación de lo necesario a posteriori, y los principios lógicos a priori son del mismo modo considerados en la determinación del valor de verdad de este tipo de enunciados .

\section{REFERENCIAS}

Alvarado, José Tomás. “La noción de justificación a priori”. Discusiones Filosóficas, 9 (12), 2008: 97-122. Impreso.

BonJour, Laurence. In Defense of Pure Reason: A Rationalist Account of A Priori Justification. Cambridge: Cambridge University Press, 1998. Print. Casullo, Albert. A Priori Justification. Oxford: Oxford University Press, 2003. Print.

Chalmers, David. "Two-Dimensional Semantics" En Lepore. E. \& Smith, B. (eds) Oxford Handbook of the Philosophy of Language. Oxford University Press, 2006: p. 574 - 606. Print.

${ }^{16}$ Énfasis de quien escribe. 
Crossley, John Newsome \& Humberstone, Lloyd. "The Logic of 'Actually'". Reports on Mathematical Logic, 8, 1977: 11-29. Print.

Fischer, Eugen \& Collins, John (eds.), Experimental Philosophy, Rationalism, and Naturalism: Rethinking Philosophical Method. Routledge. 2015. Print.

Geirsson, Heimir. "Conceivability and Defeasible Modal Justification". Philosophical Studies, 122 (3), 2005: 279-304. Print.

Gendler, Tamar \& Hawthorne, John (eds.). Conceivability and Possibility. Oxford: Oxford University Press. 2002. Print.

Howell, Robert. "The Two-Dimensionalist Reductio". Pacific Philosophical Quarterly 89 2008: 348-58. Print.

Jackson, Frank. From Metaphysics to Ethics: A Defense of Conceptual Analysis, Oxford University Press. 1998a. Print.

Jackson, Frank \& Pettit, Philip. "A Problem for Expressivism', Analysis, 58, 4, 1998b: p. 239-51. Print.

Kaplan, Rachel. The Experience of Nature: A Psychological Perspective. Cambridge University Press. 1989. Print.

Kripke, Saul. Naming and Necessity. Cambridge, MA: Harvard University Press. 1980. Print.

McLeod, Stephen. "Rationalism and Modal Knowledge". Crítica, 41 (122), 2009: p. 29-42. Print.

Mill, John Stuart. System of Logic, Ratiocinative and Inductive. En Collected Works of John Stuart Mill, Robson, J.M. (ed.), Toronto: University of Toronto Press, 1963. Print.

Miranda, Rafael. “Enunciados de identidad, invariabilidad proposicional y estipulación contextual”. Discusiones Filosóficas, 14 (23), 2013: p. 105133. Impreso.

. "Enunciados necesarios a posteriori, necesidad débil y racionalismo". Ideas y Valores, 65 (160), 2016: p. 49-74. Impreso.

. "Sobre el abuso de lo necesario a posteriori" TRANS/FORM/ AÇÃO, 40, I, 2017: p. 211-234. Impreso.

. "Rigidez de jure y de facto en los términos generales para clases naturales" Areté Vol. XXIV No 1, 2012:pp. 57 - 90. Impreso

Nimtz, Christian. (2007). Two-Dimensional Semantics - the Basics. Christian Nimtz. Web 
<http://www.unibielefeld.de/philosophie/personen/nimtz/Papers/ pbu03_Nimtz_2007_2dSemanticsLong.pdf>

Putnam, Hilary. "The Meaning of 'Meaning'". Mind, Language, and Reality. Cambridge University Press, Cambridge, 1975. Print.

Rescher, Nicholas. "Reductio ad Absurdum". Internet Encyclopedia of Philosophy (IEP), 2002. Web. <http://www.iep.utm.edu/reductio/>

Roca-Royes, Sonia. "Conceivability and De Re Modal Knowledge". Noûs 45 (1), 2011: p. 22-49. Print.

Schroeter, Laura. "Two-Dimensional Semantics". The Stanford Encyclopedia of Philosophia, Zalta, E. (ed.), 2012. Web <http://plato. stanford.edu/archives/win2012/entries/two-dimensional-semantics/> Schwartz, Stephen. "Mill and Kripke on Proper Names and Natural Kind Terms". British Journal for the History of Philosophy, 21 (5), 2013: p. 925-945. Print.

Soames, Scott. "Kripke on Epistemic and Metaphysical Possibility". Saul Kripke, Berger, Alan. (ed.), Cambridge University Press. 2011. Print.

Stalnaker, Robert. "Possible Worlds". Nous 10, 2013: p. 65-75. Print.

Tahko, Tuomas. "On the Modal Content of a Posteriori Necessities". Theoria, 75, 2009: p. 344-357. Print.

Tahko, Tuomas. "A Priori and A Posteriori: A Bootstrapping Relationship". Ontology Metaphysics, 12, 2011: 151-164. Print.

Yablo, Stephen. "Concepts and consciousness". Philosophy and Phenomenological Research, 59 (2), 1999: p. 455-463. Print. 